\title{
Special issue on advances in nature inspired algorithms for complex numerical optimization problems
}

\author{
Jagdish Chand Bansal • Millie Pant • Kusum Deep • \\ Harish Sharma
}

Published online: 9 November 2013

(C) Springer-Verlag Berlin Heidelberg 2013

\section{Introduction}

Optimization problems occur in almost all spheres of human activities. However, with the increasing competitiveness in the real world scenario, the mathematical models of such problems are getting more and more complex. In fact, cases may appear where it become difficult to place a problem under a specific category. In order to deal with such cases, sophisticated techniques/algorithms are needed. Nature has always been a source of inspiration for researchers to develop algorithms for the complex numerical optimization problems which are usually infeasible to solve using traditional techniques. The computational algorithms inspired from nature fall under the category of Nature Inspired Algorithms (NIA). In the past few years, researchers have given much attention to NIA which not only give a reasonably good solution but are also computationally fast. Some of the popular NIA for complex numerical optimization include genetic algorithms, simulated annealing, differential evolution, particle swarm optimization, bacterial foraging optimization, artifi-

\section{J. C. Bansal (凶)}

South Asian University, New Delhi, India

e-mail: jcbansal@gmail.com

Present address:

J. C. Bansal

South Asian University, New Delhi, India

\section{Pant $\cdot$ K. Deep}

Indian Institute of Technology Roorkee, Roorkee, India

e-mail: millidma@gmail.com

K. Deep

e-mail: kusumfma@iitr.ernet.in

H. Sharma

Government Engineering College Jhalawar, Jhalawar, Rajasthan, India e-mail: harish0107@rediffmail.com cial bee colony, neural networks, and quantum computing. The five papers included in this Special Issue are selected after a rigorous review process and at least two review rounds. Large number of high quality submissions to this Special Issue, clearly reflects the strong interest of researchers in the field of NIA.

\section{Contributions}

This special issue contains total five research articles in different areas of nature inspired algorithms. In the paper by Antonio LaTorre, Santiago Muelas and Jose-Maria Pena, Evaluating the Multiple Offspring Sampling Framework on Complex Continuous Optimization Functions, a hybrid HRH Dynamic algorithm is presented. The hybrid algorithm has been constructed by using the multiple offspring sampling framework. The algorithm is obtained by hybridizing two very successful algorithms, differential evolution and IPOPCMA-ES. The proposed algorithm combines the explorative and exploitative capabilities of the two algorithms. The proposed algorithm is well tested over noisy and noiseless test functions.

Next paper is Distributed Mixed Variant Differential Evolution Algorithms for Unconstrained Global Optimization by G. Jeyakumar and C. Shunmuga Velyautham which proposes a novel variant of differential evolution. The proposed algorithm is named as Distributed Mixed Variant Differential Evolution (dmvDE). The novelty of dmvDEs lies in mixing different $\mathrm{DE}$ variants in an island based distributed framework. dmvDE employ four effective DE variants: DE/rand/1/bin, DE/rand/2/bin, DE/best/2/bin and DE/randto-best/1/bin with diverse characteristics in various proportions and in various combinations. In this way, total 19 dmvDE algorithms are proposed. All $19 \mathrm{dmvDEs}$ are tested over 14 unconstrained test bed. 
In the paper, K-page Crossing Number Minimization Problem: An Evaluation of Heuristics and its Solution using GESAKP by Dharna Satsangi, Kamal Srivastava and Gursaran, a guided evolutionary simulated annealing (GESAKP) is presented for the $\mathrm{K}$-page crossing minimization problem (KPMP). Extensive experiments were carried out on standard and random graphs to assess the performance of GESAKP. From the experimental results, authors also presented the conjectures for the K-page crossing number of some complete tripartite graphs and page number of toroidal meshes and a class of complete bipartite and tripartite graphs. Overall, the paper establishes the applicability of GESAKP for KPMP.

Fourth paper, Design and Simulation of FIR Band Pass and Band Stop Filters using Gravitational Search Algorithm by Suman Kumar Saha, Rajib Kar, Durbadal Mandal and Sakti Prasad Ghoshal, proposes a new optimization method, Gravitational Search Algorithm (GSA). In the paper, problem of the constrained, multi-modal, non-differentiable, and highly nonlinear FIR band pass and band stop filter design is dealt with GSA. Results for the problem are obtained by GSA and compared with that of real coded genetic algorithm, particle swarm optimization (PSO), differential evolution, bee swarm optimization.

The last paper, Bi-level Thresholding Using PSO, Artificial Bee Colony and MRLDE embedded with Otsu Method by Sushil Kumar, Pravesh Kumar, Tarun Kumar Sharma and Millie Pant, proposes a hybrid method for image thresholding based on the hybridization of MRLDE and Otsu method. MRLDE+Otsu is tested over a test bed of ten images and the results are compared with PSO+Otsu and $\mathrm{ABC}+\mathrm{Otsu}$.

Acknowledgments Guest editors would like to thank all the people that have contributed to this Special Issue. In particular, we thank the Managing Editor-in-Chief, Meng-Hiot Lim and Technical Editors-inChief Steven Gustafson, Natalio Krasnogor and Yew Soon Ong for their support and help during the process. Thank you to the numerous referees for their expertise and all the hard work allocated to the reviewing task. 